\title{
Global Suicide Rate Among Youngsters Increasing Significantly
}

\author{
Bahman Zohuri $^{1 *}$ and Siamak Zadeh ${ }^{2}$ \\ ${ }^{1}$ Electrical Engineering and Computer Department, University of New Mexico, New Mexico, USA \\ ${ }^{2}$ Golden Gate University, Ageno School of Business, San Francisco, USA
}

*Corresponding author: Bahman Zohuri, Electrical Engineering and Computer Department, University of New Mexico, Albuquerque, New Mexico, USA

\section{Abstract}

Suicide is a key health concern and a major cause of death among persons aged $10-24$ years in the United States. In 2017, suicide was the second leading cause of death among this group and from 2007 to 2018, the suicide rate increased $56 \%$ among those aged 14 - 19 years. Overall, among teenagers 12 - 19 years, suicide is the third leading single cause of death, after accidents (unintentional injuries) and homicides. According to the latest World Health Organization (WHO), suicidal behavior is also a major health concern in many countries, developed and developing alike. Nearly 800,000 are estimated to die annually from suicide worldwide. Many more, particularly the young and the middle-aged individuals, attempt suicide. In this paper, it is suggested that modern technology plays a significant role in the increase in the rate of suicide among the youths. It is suggested that increasing engagement with social media, driven by new generation of electronic gadgets and smart phones, is an important factor behind the increase in the rate of suicides. Heavy usage of smart phones, such as texting, twitting, or having some other forms of engagement through social media, is a contributing factor.
\end{abstract}

Keywords: Depression; Suicide Attempt and Suicide Rate; Youngsters; Suicide Risk Management; Augmentation of Artificial Intelligence in Depression Treatment

\section{Introduction}

Suicide is a major public health problem and a leading cause of death among the persons $10-24$ years in the United States. The effects of suicide go beyond the person who acts to take his or her life. It can also have a lasting effect on family, friends, and the public at large. The suicide attempts among youth and youngsters between age of 14 to 30 , globally has been increasing in recent years. According to the statistics, there is a shocking rise in suicide attempts among youngsters. In fact, between 2000 and 2007, the suicide rate among youth ages 10 to 24 hovered around 6.8 deaths per 100,000 people. Then, the rate curved upward, reaching a rate of 10.6 deaths per 100,000 by 2017 - a 56-percent increase in less than two decades. The suicide rate among people ages 10 to 24 years old climbed 56\% between 2007 and 2017, according to the report from the Centers for Disease Control and Prevention. The rate of homicide deaths decreased by 23\% from 2007 to 2014 but then increased by $18 \%$ through 2017 [1].
Violent death, including homicide and suicide, is a major cause of premature death for the age group. Around 2010, the death rate of suicides among adolescents and young adults surpassed the rate of homicide deaths, according to the report. Suicide was the secondleading cause of death among 10- to 24-year-olds in 2016, up from third place in earlier years, according to the Centers for Disease Control (CDC) and Prevention [1].In this context, the researchers are calling for more study of possible gender differences in youth mental health, noting previous findings of a larger rise in depression in teenage girls compared with boys in the decade leading up to 2014. One of the main and major cause of suicide attempt among is the Impact of "Technology on Mental Health" among these youths $[2,3]$.

In a nutshell, Mental health refers to our cognitive, behavioral, and emotional wellbeing - it is all about how we think, feel, and 
behave. The term 'mental health' is sometimes used to mean an absence of a mental disorder. Neurological disorders with symptoms such as chronic pain, depression, and insomnia are widespread. Very weak electric fields applied through the skull can enhance or diminish neural activity and modulate brain waves in order to treat many of these common medical problems. This approach is to be contrasted with well-established pharmacological methods or more recent invasive electrical Deep Brain Stimulation (DBS) techniques that require surgery to insert electrodes deep into the brain. We claim that Non-Invasive Brain Stimulation (NIBS) will provide new treatment methods with much greater simplicity, lower cost, improved safety and in some cases, possibly greater effectiveness

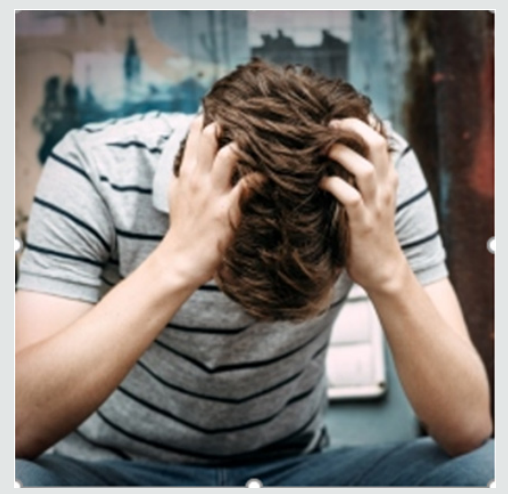

Figure 1: Isolation and Silo Daily Life.

Mental health can affect daily life, relationships, and even physical health. Mental health also includes a person's ability to enjoy life - to attain a balance between life activities and efforts to achieve psychological resilience [4].One of the side effects of modern technology and our dependency on electronic gadget is isolation and living in a silo mode among the youths and being member of a social media is like adding salt to injury as far as we are concerned (Figure 1). Furthermore, Neurological disorders with symptoms such as chronic pain, depression, and insomnia are widespread among "Global Population" in particular among youngsters in more developed countries and the fast paste life that these youths are living in. Very weak electric fields applied through the skull can enhance or diminish neural activity and modulate brain waves in order to treat many of these common medical problems. This approach is to be contrasted with well-established pharmacological methods or more recent invasive electrical Deep Brain Stimulation (DBS) techniques that require surgery to insert electrodes deep into the brain. We claim that Non-Invasive Brain Stimulation (NIBS) will provide new treatment methods with much greater simplicity, lower cost, improved safety and in some cases, possibly greater effectiveness. This emerging use of NIBS is a branch of a new multidisciplinary field that we coined Neuro-systems Engineering [5]. This field involves neuroscientists, psychologists, and electrical engineers. This emerging field relies on existing standards for the safe implementation of these novel treatment modalities. Methods of stimulating the brain are based on emerging electro-technologies such as transcranial [6]. As summary of this introduction, we can express that, Depression is a serious medical illness. It's more than just a feeling of being sad or "blue" for a few days. If you are one of the more than 19 million teens and adults in the United States who have depression, the feelings do not go away. They persist and interfere with your everyday life [7].

Symptoms can include:

- Feeling sad or "empty"

- Loss of interest in favorite activities

- Overeating, or not wanting to eat at all

- $\quad$ Not being able to sleep, or sleeping too much

- $\quad$ Feeling very tired

- $\quad$ Feeling hopeless, irritable, anxious, or guilty

- $\quad$ Aches or pains, headaches, cramps, or digestive problems

- Thoughts of death or suicide

Depression is a disorder of the brain. There are a variety of causes, including genetic, biological, environmental, and psychological factors. Depression can happen at any age, but it often begins in teens and young adults. It is much more common in women. Women can also get postpartum depression after the birth of a baby. Some people get seasonal affective disorder in the winter. Depression is one part of bipolar disorder.

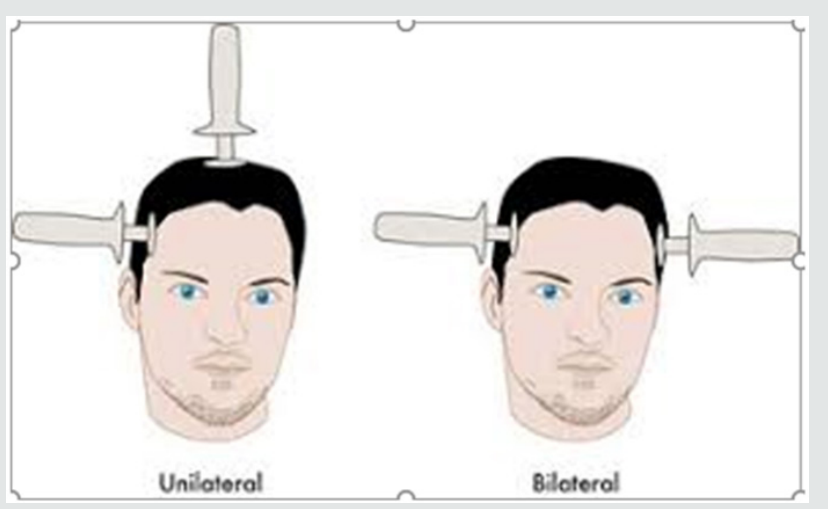

Figure 2: Electroconvulsive Therapy (ECT).

There are effective treatments for depression, including antidepressants, talk therapy, or both. Although the common treatment of severe depression is the hospitalization these types of patients following series electrical shock therapy such as Electroconvulsive Therapy (ECT) highly recommended by phycologist and physiatrist these days, which is very invasive approach, yet there are other suggested treatment that may have similar resultyet not being as invasive as ECT. As (Figure 2) illustrates two electrode probe is used to send electrical shock to patient brain 
and side effect of beside putting patient under anesthesia, in a long would cause permanent lapse of patient memory which is very common among patients going through this procedure. However, these authors do suggest another way of treating depression using a non-invasive approach such as Transcranial Magnetic Stimulation (TMS) or Transcranial Electric Stimulation (TES) or any other aspect of these types of treatments such as $r$ TMS or $r$ TES that are standing for repetitive TMS or TES respectfully [2,3]. There are mind research centers that are seriously looking for this noninvasive treatment these days and their data shows a good evidence of a positive and conclusive results.

\section{Who is at Risk for Suicide and Why?}

According to the MedlinePlus web site [8], suicide does not discriminate. It can touch anyone, anywhere, at any time. But there are certain factors that can contribute to the risk of suicide, including:

- Having attempted suicide before

- Depression and other mental health disorders

- Alcohol or drug use disorder

- Family history of a mental health disorder

- Family history of an alcohol or drug use disorder

- $\quad$ Family history of suicide

- Family violence, including physical or sexual abuse

- Having guns in the home

- Being in or having recently gotten out of prison or jail

- $\quad$ Being exposed to others' suicidal behavior, such as a family member, peer, or celebrity

- Medical illness, including chronic pain

- Stressful life event, such as a job loss, financial problems, loss of a loved one, a breakup of a relationship, etc.

- $\quad$ Being between the ages of 15 and 24 years or over age 60

Suicide is a major cause of death among youths as we stated at the beginning of this article is on exponential rise and it is becoming a serious threat to our future national brain investment of our youths and has such devesting on the family that are losing their youngsters to such episode. (Figure 3) is indication of American youngsters and young adults, that are victim of suicide that is seriously drive by depression scenario and it is leading single cause of death, after accidents and homicide. "The chances of a person at the young age range dying by suicide are greater than homicide, when it used to be the reverse," said Sally Curtin, a statistician at the CDC and an author of the report. "When a leading cause of death among our youth is increasing, it behooves all of us to pay attention and figure out what's going on".
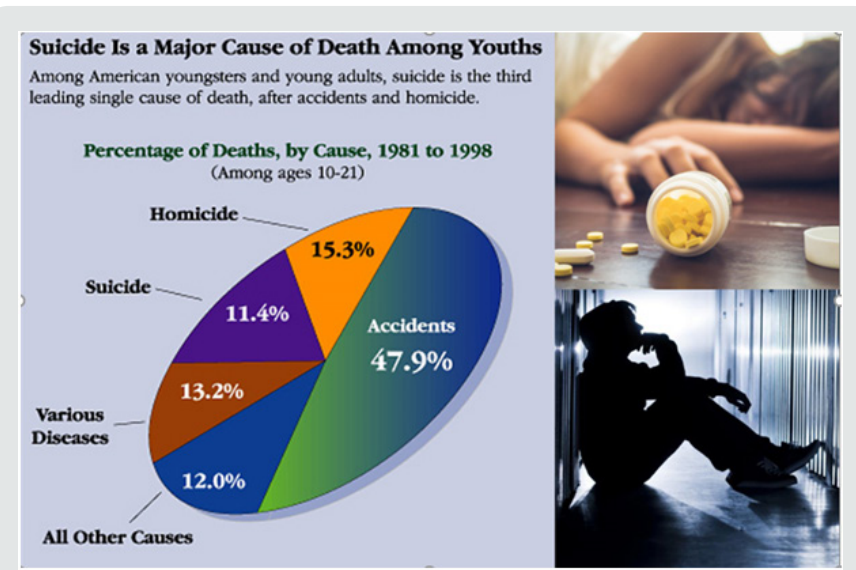

Figure 3: Suicide Is a Major Cause of Death Among Youngs.

Suicide rates in general have increased [8] in the U.S. across all ages and ethnic groups, rising roughly 30\% from 1999 to 2016. In 2017, suicide was the second-leading cause of death among those ages 10 to 24, behind unintentional injuries, such as car crashes or drug overdoses (Figure 4). Suicide death rates have risen significantly in most states since the late 1990s, with 25 states recording increases of more than 30\%, the Centers for Disease Control and Prevention said. The rates rose in men and women and across all age and ethnic groups, propelled by mental illness, substance-use disorders, financial hardship and relationship problems, the CDC said. Death by firearm was the most common method of suicide, accounting for $48.5 \%$ of cases.

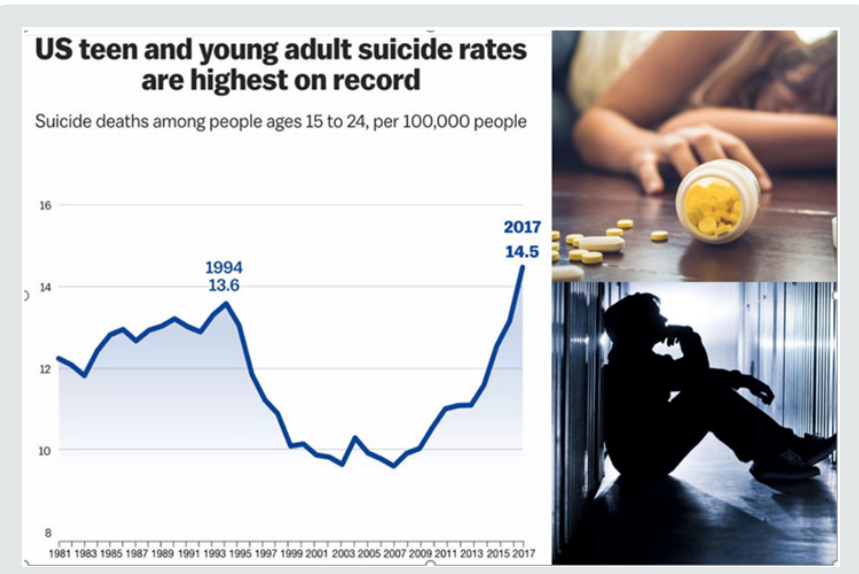

Figure 4: US Teen and Young Adult Suicide Rates Are Rising.

The data that is pulled from death-certificate data from CDC National Vital Statistics System, looking at the underlying cause of death for people ages 10 to 24 . They analyzed data from 2000 to 2017 , the most recent year of CDC's available data. Both suicide and homicide deaths among the age group were relatively stable from 2000 to 2007, the report says [1]. Within the next few decade, suicide deaths increased from 6.8 deaths per 100,000 people to 10.6 deaths, with 2,449 more suicides in 2017 than in 2007 time period. While the youth in the ages between 10- to 14-year-olds 
had by far the lowest rate of suicides, that rate nearly tripled from 2007 to 2017 [1].

"Unfortunately, it's not surprising, but it is highly disturbing," said Benjamin Shain, a child and adolescent psychiatrist at NorthShore Medical Group in Illinois, who says he is increasingly seeing adolescent patients at risk for suicide. "To see it statistically across the country hits me in a different way." Homicide deaths ranked third, according to a CDC report from June 2019. Despite concern over the rising suicide rates, researchers aren't sure of the exact causes. A rise in depression among adolescents, drug use, stress and access to firearms might all be contributing factors, experts say.

Some mental-health experts suggest that social-media use among teens might be fueling the increase in mental-health conditions and leading to greater suicide risk, and some early studies have linked smartphone use to anxiety, depression and sleep deprivation among adolescents. The recent visibility of suicide in the media and online might also increase suicide death rates, experts say. (Figure 5) obtained from the article reported by Wasserman et. al. and quoted from the latest World Health Organization (WHO) is good indication of suicide rate in 26 countries (areas), data that were available for the whole period studied between period of 1965 - 1999 and they are broken by gender and expended during three periods (1965-1979, 1980-1989 and 1990-1999).

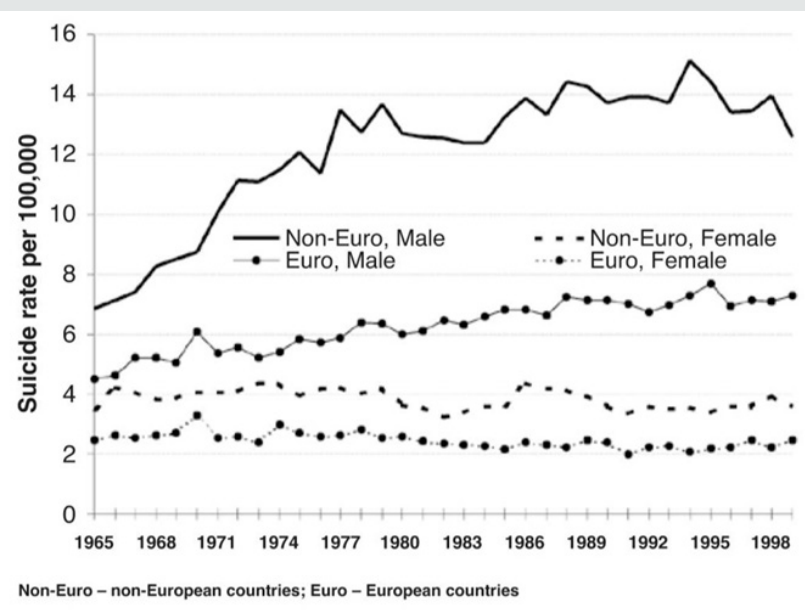

Figure 5: Suicide Rate Among 26 Countries.

Suicidal behavior is a major health concern in many countries, developed and developing alike. At least a million people are estimated to die annually from suicide worldwide [9] Many more people, especially the young and middle-aged, attempt suicide [10]. Some worldwide analyses of suicide trends and rates in the world have been published [11-14] but very little is known worldwide about the causes of death and suicide rates among young people aged 15-19. International comparability of data is also discussed in these refences. The information used is reflecting the official figures reported to WHO by member states, is based on death certificates signed by legally authorized personnel - usually doctors or police officers in the respective country. Usually these professionals have specific routines. How these routines differ between countries and regions, and how they influence suicide statistics, remains to be demonstrated through comparative studies of mortality statistics [1].

\section{What are the Warning Signs for Suicide?}

Some people may tell others about their suicidal thoughts. But others may try to hide them. This can make some of the signs harder to spot [8].

\section{The warning signs for suicide include:}

- $\quad$ Talking about wanting to die or wanting to kill oneself

- Making a plan or looking for a way to kill oneself, such as searching online

- $\quad$ Buying a gun or stockpiling pills

- $\quad$ Feeling empty, hopeless, trapped, or like there's no reason to live

- $\quad$ Being in unbearable pain

- $\quad$ Talking about being a burden to others

- $\quad$ Using more alcohol or drugs

- Acting anxious or agitated, behaving recklessly

- Sleeping too little or too much

- Withdrawing from family or friends or feeling isolated

- Showing rage or talking about seeking revenge

- $\quad$ Displaying extreme mood swings

- $\quad$ Saying good-bye to loved ones, putting affairs in order

One of the initial approaches by anyone who is feeling depressed, is that she or he should stay connected rather than staying in a total isolation and continue with silo day-to-day life. As a friend, member of family, co-worker etc. we should ask the person if they are thinking about killing themselves or keep them safe by finding out whether they have a plan for suicide and keep them away from things that they can use to kill themselves that even may include keep them away from their electronic gadget such as Smart Phone as well as social media and Internet as part of driven factor by modern technology.

To be there with them, when they need us and listen carefully and find out what are they thinking and feeling and finally helped them connected to resources that can help them, such as: 
- $\quad$ Calling the National Suicide Prevention Lifeline at 1-800273-TALK (1-800-273-8255). Veterans can call and press 1 to reach the Veterans Crisis Line.

- $\quad$ Texting the Crisis Text Line (text HOME to 741741)

- $\quad$ Texting the Veterans Crisis Line at 838255

These are very important steps that people around youngsters can do to help them by keeping them away from their suicide thoughts and commitment to it.

\section{The Impact of Technology on Mental Health}

As we have stated at the beginning, mental health can affect daily life, relationships, and even physical health. Mental health also includes a person's ability to enjoy life - to attain a balance between life activities and efforts to achieve psychological resilience [4]. "Emotional, behavioral, and social maturity or normality; the absence of a mental or behavioral disorder; a state of psychological well-being in which one has achieved a satisfactory integration of one's instinctual drives acceptable to both oneself and one's social milieu; an appropriate balance of love, work, and leisure pursuits".

As it can be observed in (Figure 6), the mental health problems have no age limit and can affect anyone at any age and statics shows a growing epidemy among youths in globally driven by fast paste modern technology and world of electronic and gadgets.

According to the World Health Organization (WHO), mental health is "...a state of well-being in which the individual realizes his or her own abilities, can cope with the normal stresses of life, can work productively and fruitfully, and is able to make a contribution to his or her community". Experts say we all have the potential to develop mental health problems, no matter how old we are, whether we are male or female, rich or poor, or which ethnic group we belong to.

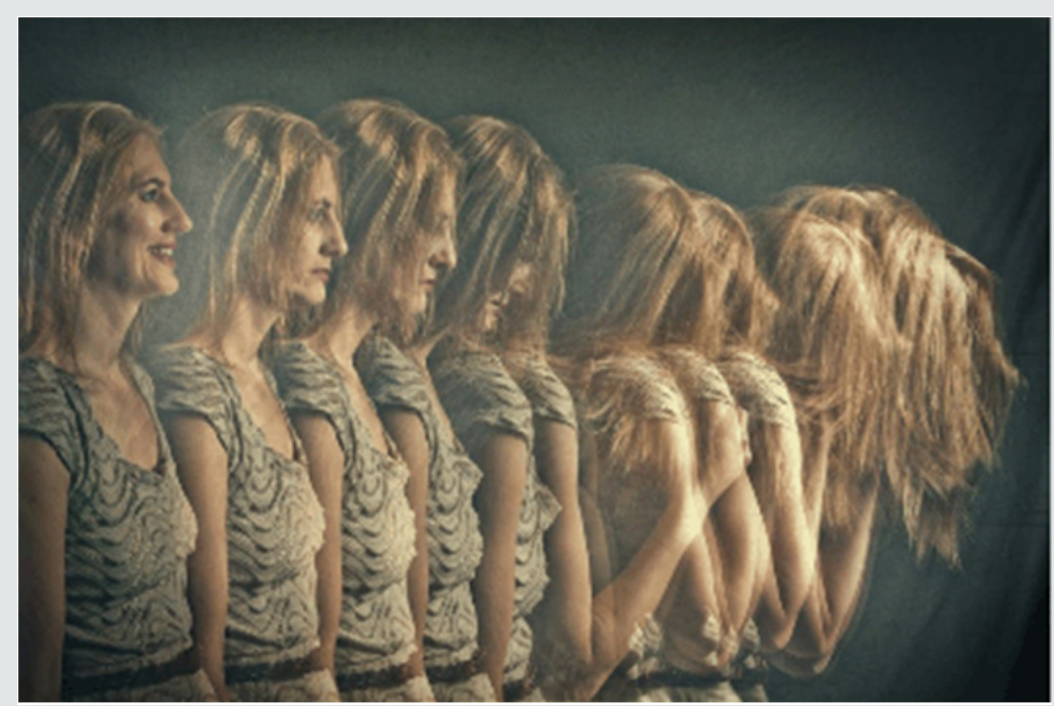

Figure 6: Impact of Technology on People.

Almost 1 in 5 Americans experiences mental health problems each year (18.5 percent). In the United States, in 2015, an estimated 9.8 million adults (over 18) had a serious mental disorder. That equates to 4.8 percent of all-American adults. A large proportion of the people who have a mental disorder have more than one. In the U.S. and much of the developed world, mental disorders are one of the leading causes of disability. Mental illnesses are common in the United States. Nearly one in five U.S adult's lives with a mental illness (44.7 million in 2016). Mental illnesses include many different conditions that vary in degree of severity, ranging from mild to moderate to severe. Two broad categories can be used to describe these conditions: Any Mental Illness (AMI) and Serious Mental Illness (SMI). AMI encompasses all recognized mental illnesses. SMI is a smaller and more severe subset of AMI. Additional information on mental illnesses can be found on the National Institute of Mental Health (NIMH) Health Topics Pages [15].
Note that The National Institute of Mental Health (NIMH), the lead federal agency for research on mental disorders, offers basic information on mental disorders, a range of related topics, and the latest mental health research. It is not the intention of NIMH to provide specific medical advice, but rather to provide users with information to better understand their health and their diagnosed disorders. Consult with a qualified health care provider for diagnosis, treatment, and answers to your personal questions.

Dealing with the subject of this section, one important question that we need to ask is How modern life affects our physical and mental health given fast paste growth of modern technology and increasing volume of information that brain needs to process? [16] Modern day living is a multifaceted compendium of evolving technology and social media. Communication outlets are changing every part of our lives so rapidly that it can be tough to adjust. Are technology and media affecting our physical and mental health? 
See (Figure 7). Technology has improved the lives of many people, with almost half of adults in the United States unable to imagine life without their smartphone.

As (Figure 8) is illustrating, the technological and social media advances of the past decade have taken over our lives. As this illustration presents, today's vast volume of data and consequently information has tremendous impact on day-to-day life of folks to the point that we can observe everyone is on their Personal Device Assistant (PDA) at any moment of their life. Do they affect our physical and mental health? The American Psychological Association's Stress in America Survey 2017 shows that 99 percent of adults own an electronic device, around 86 percent own a computer, 74 percent own a smartphone, and 55 percent own a tablet.

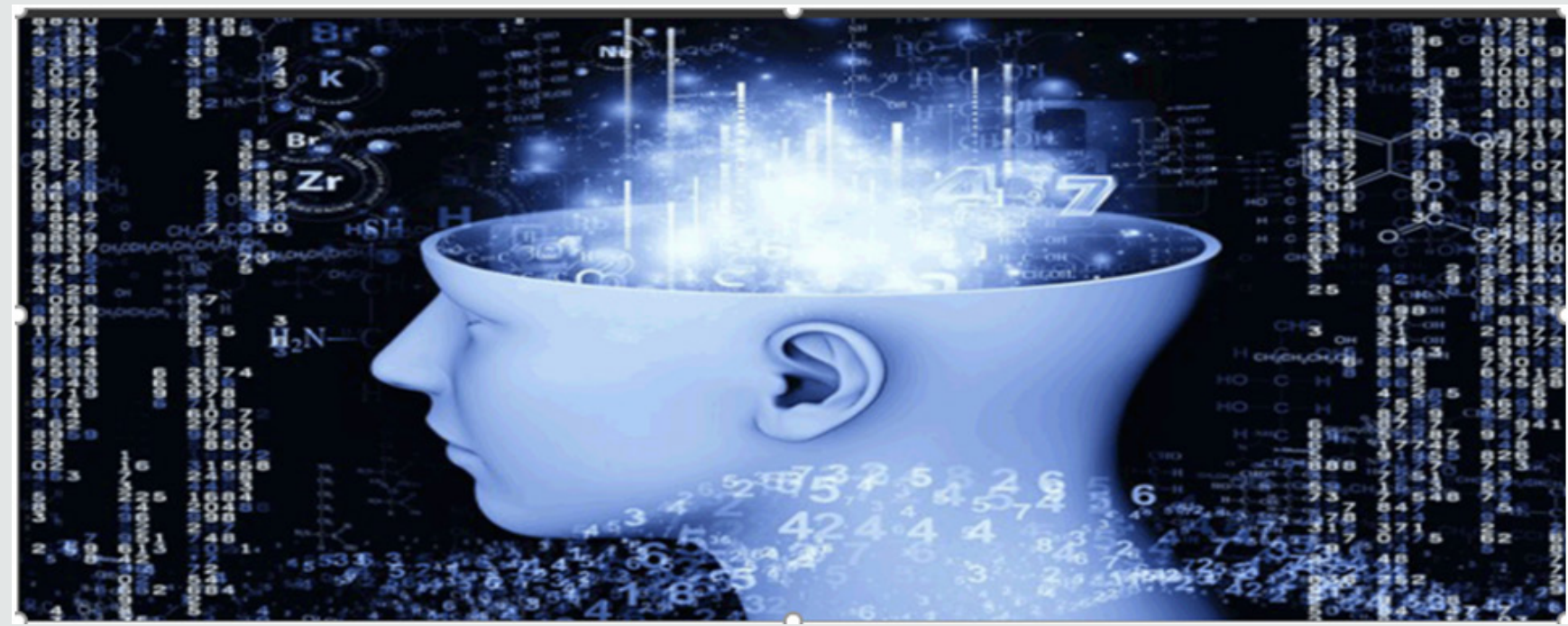

Figure 7: Illustration of Brain Activities Driven by Internet of Thing (IoT).

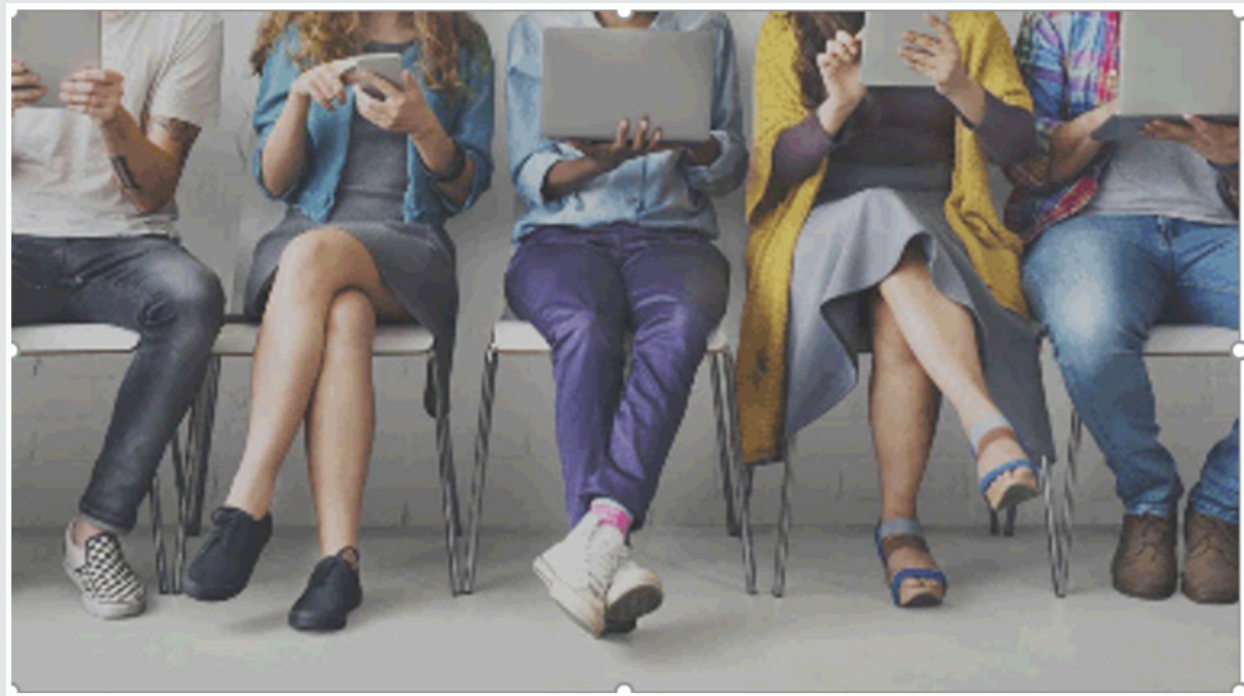

Figure 8: People Drive by Electronic Gadgets.

The survey also reports that between 2005 and 2015, the percentage of adults using social media skyrocketed from 7 percent to 65 percent, with usage rates of young adults aged between 18 and 29 increasing from 12 percent to 90 percent in that period. Rates of technology and social media use are therefore swiftly climbing. Facebook and Instagram alone boast a combined monthly user base of 2 billion people. Recent research by The Associated Press-NORC Center for Public Affairs Research found that teenagers aged 13 to 17 years old have shifted their preferred social media platforms and are now most likely to use Snapchat and Instagram.

Key findings of the survey included the fact that around 76 percent of teenagers use Instagram, 75 percent use Snapchat, 66 percent use Facebook, 47 percent use Twitter, and fewer than 30 percent use Tumblr, Twitch, or LinkedIn. They also found that although 91 percent of teens use regular text messaging, 40 percent also use messaging apps such as WhatsApp, Kik, or Line. 
Social media and text messaging have become an integral part of how individuals interact with their social groups. In fact, for many teenagers and young adults, text messaging and social media communication is now more likely than in-person interactions.

The more details about this issue can be chapter 4 of the book by Zohuri [2] and we strongly recommend the readers of this article to refer to this reference as well. Furthermore, while many people strongly agree that unplugging or taking a digital detox now and then is important for mental health, in reality, only 28 percent of those people periodically switch off from technology. Across the generations, 48 percent of Millennials, 37 percent of Gen Xers, 22 percent of Boomers, and 15 percent of Matures are worried about the negative effects of social media on their physical and mental health.

As (Figure 9) indicates using social media for extended periods is associated with depression. Interactions on social media can have a major impact on an individual's well-being and satisfaction. Many studies have observed that more time spent on social media is associated with an increased risk of loneliness and depression, which poses the question: are unhappy people using social media, or does social media use affect happiness?

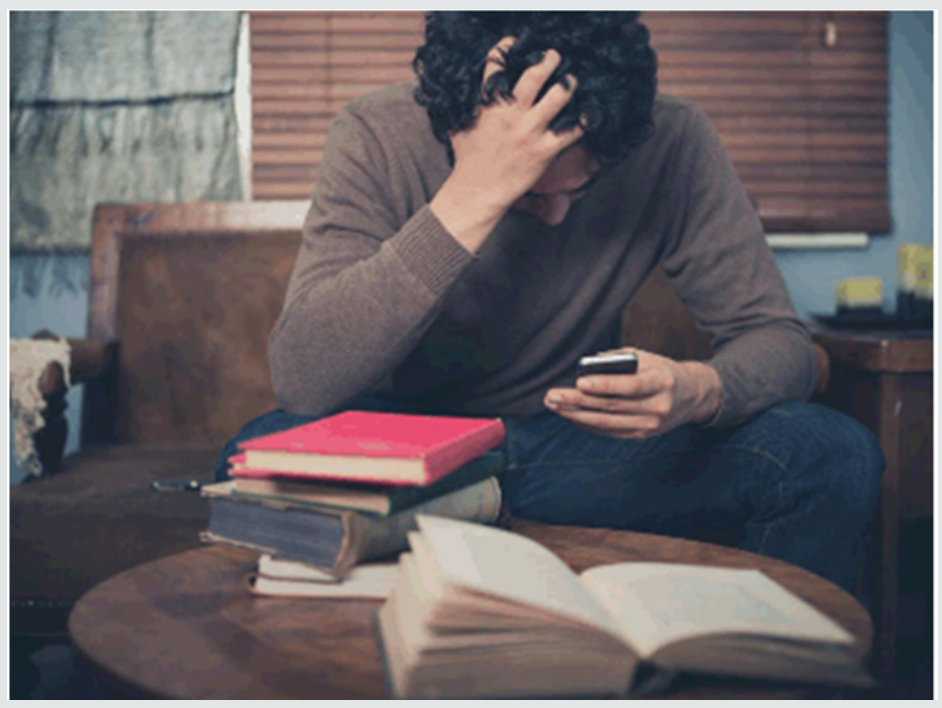

Figure 9: Using Social Media for Long Period of Time.

\section{Transcranial Magnetic Stimulation Driving Depression Treatment}

As we start introducing Transcranial Magnetic Stimulation (TMS) as most effective and non-invasive way of treating at least mild-depression along with other branches of this technology such as RTMS, TES and RTES, we need to present a holistic description of TMS approach for those readers that do not have any background such technological background what is the TMS. Transcranial magnetic stimulation (TMS) is a noninvasive method to excite neurons in the brain: weak electric currents are induced in the tissue by rapidly changing magnetic fields (electromagnetic induction) [16] This way, brain activity can be triggered with minimal discomfort, and the functionality of the circuitry and connectivity of the brain can be studied.

The principle of inductive brain stimulation with eddy currents has been noted since the 19th century. The first successful TMS study was performed in 1985 by Anthony Barker [17] in Sheffield, England. By stimulating different points of the cerebral cortex and recording responses, e.g., from muscles, one may obtain maps of functional brain areas. By measuring functional imaging (e.g.
Magnetic Resonance Imaging (MRI)) or Electroencephalogram (EEG), where this test records the electrical signal of the brain and the information may be obtained about the cortex (its reaction to TMS) and about area-to-area connections.

The basic principle of Transcranial Magnetic Stimulation is shown schematically in (Figure 10), where TMS is showing a timevarying pulse of current in an external coil causing inducing currents in the brain. Magnetic stimulation can be used as an alternative to conventional electrical stimulation of nerves in some applications because it has a number of advantages which are discussed later. Applications include deep peripheral nerve stimulation and the non-invasive and painless stimulation of the human brain, both to elicit responses directly and to modify excitability and plasticity. Magnetic stimulation can be used as an alternative to conventional electrical stimulation of nerves in some applications because it has a number of advantages which are discussed later. Applications include deep peripheral nerve stimulation and the non-invasive and painless stimulation of the human brain, both to elicit responses directly and to modify excitability and plasticity. Repetitive TMS stimulation is known as RTMS and can produce longer lasting 
changes. Numerous small-scale pilot studies have shown it could be a treatment tool for various neurological conditions (e.g. migraine, stroke, Parkinson's disease, dystonia, tinnitus) and psychiatric conditions (e.g. major depression, auditory hallucinations). TMS is becoming more widely used for treating nonresponsive severe depression. (Figure 11) shows how the TMS coil produces that fields, where noninvasively interact with the brain. In this figure, the time-changing magnetic field (Red Color) induces the electric field and currents (Green color) in the brain. (Courtesy E.M. Wassermann) and current in the coil (Yellow color).

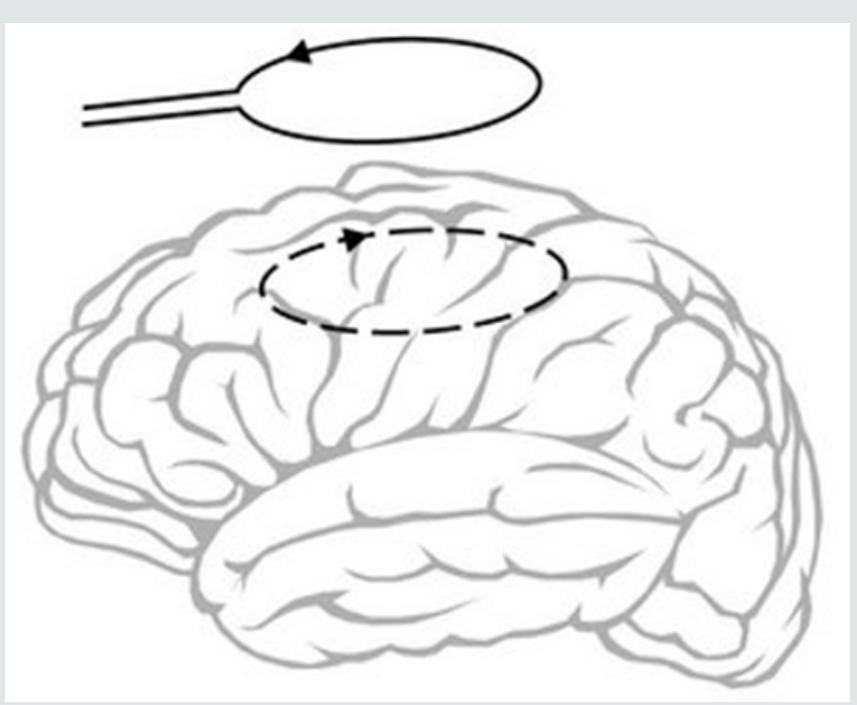

Figure 10: The Basic Principle of Transcranial Magnetic Stimulation Depiction.

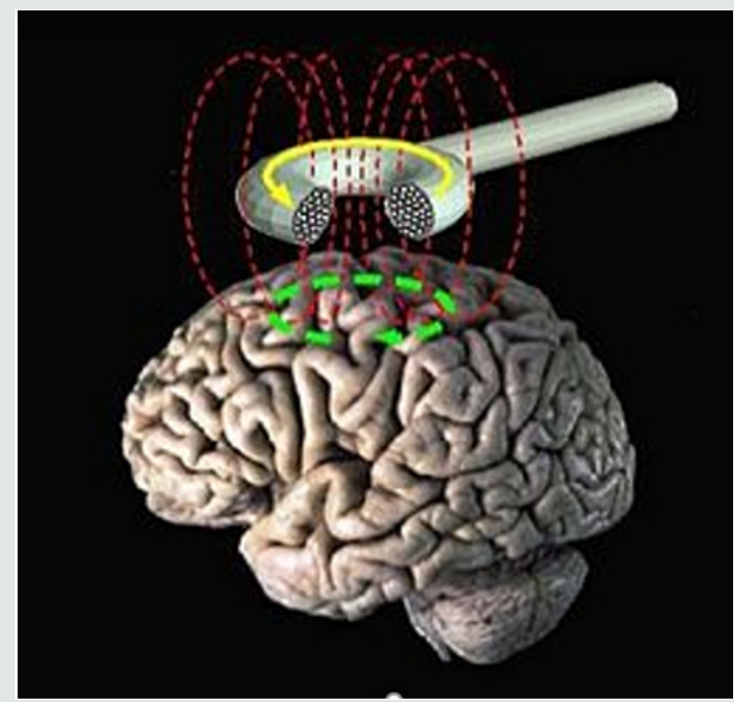

Figure 11: A TMS Coil Suspended Over the Brain.

In the last 20 years, the use of Non-Invasive Electrical Brain Stimulation (NIBS) has transitioned from the ad hoc use of a variety of techniques and methods to a more system engineering approach to achieving desired methods to treat neurological disorders tailored to specific problems and individual characteristics [18]. We have described this approach to the combination of neuroscience, psychology, and electrical engineering as the new field of Neuro systems Engineering [19] We believe this new multidisciplinary engineering approach will have a profound and lasting impact on serious health problems. Our specific and original concept is a systematic combination of methods described in the following sub-sections, TACS, TRNS, and real time control to achieve major cognitive benefits. This is to be compared with prescriptive application of NIBS, or the use of pharmacological prescriptions that treat disease, but create undesirable outcomes: neither are individualized to the disorder or the individual except through a process of trial and error. We are proposing an approach based on treating specific problems from a Neuro system Engineering point of view.

A Neuro systems Engineer approaches the solution to problems through first focusing on the specific problem, and then applying 
the scientific method of hypothesis, experimentation, data collection, and modification of the process and the hypothesis until a consistent explanation is achieved in order to solve the problem. We believe this approach resulted in our thinking about tailored control to focus the dose, duration, frequency spectrum, and electrode geometry to deal with the individual and the disorder. Individualized treatment approaches, using neuroimaging, are beginning to be used in the field of ECT [20] and we believe that this approach can be applied more broadly and with a high degree of fidelity.

\section{Transcranial Alternating Current Stimulation (TACS)}

Relatively few studies of transcranial Alternating Current Stimulation (TACS) have been undertaken, a technique designed to change intrinsic cortical oscillations. The current can be applied to the earlobes or the sides of the head, known as Cranial Electrotherapy Stimulation - (CES) [21]. This technique opens up the possibility of actively synchronizing cortical rhythms through external means particularly when administered in the so-called "ripple" frequency range (between 100 and $250 \mathrm{~Hz}$ ) associated with memory encoding [22]. This technique must be used at relatively low frequencies or retinal flashes will be induced; moreover, at higher amplitudes, safety concerns - including seizures - remain. In spite of these very particular reservations, active synchronization of cortical oscillatory activity provides particularly enticing possibilities regarding very fine-tuned experiments involving stimulation, behavior modification, recording of neuronal activity, and alteration of stimulation in real time. Currently, the TACS method is primarily used to treat depression, anxiety, and other mood related disorders.

\section{Transcranial Random Noise Stimulation (TRNS)}

The most recent player to the electrical stimulation field is transcranial Random Noise Stimulation (TRNS). This method, akin to adding "white noise" to ongoing neural activity, is thought to open ion channels at the neuron [23] In one of the first studies in normal human subjects, stimulation over the motor cortex (between 100 and $640 \mathrm{~Hz}$ for 10 minutes at $1.5 \mathrm{mAmp}$ ) was found to increase cortical excitability [24] These authors hypothesize that, like TACS, TRNS "can possibly interfere with ongoing oscillations and neuronal activity in the brain and thus result in a cortical excitability increase."

\section{There are several potential advantages of TRNS over TDCS:}

a. While Transcranial direct current stimulation (TDCS) can open ion channels once, TRNS can do so repeatedly through multiple ionic influxes.

b. TRNS works around problems associated with stimulation of different sides of a folded cortex which can lead to effects which cancel each other out; c. TRNS does not create a "tingling" sensation, as does TDCS when applied;

d. safety concerns are minimized.

A recent study found superior performance of high frequency $(100-640 \mathrm{~Hz})$ tRNS over tDCS (1.5 mAmp) in normal subjects on a perceptual learning task [25] A second study did not find improved performance of TRNS over TDCS in normal subjects on a working memory paradigm [26] Only a handful of studies have been undertaken with TRNS; however, this technique appears to combine the main benefits of both TDCS (e.g., ionic influx/neuronal excitation) and TACS (e.g., entrainment of oscillations) without many of the potential downsides (e.g., burning, retinal stimulation, possible seizures, etc.).

In conclusion for the above methodology of treatment depression and brain disorder, we believe that this Neurosystems Engineering approach will provide improved understanding of many neurological disorders and the ability to achieve real improvements in a nonpharmacological, painless, low cost, and practical approach that will have an enduring impact on society. Future advances in this electrotechnology must be achieved in coordination with the standards community.

\section{Feasibility of Artificial Intelligence in Suicide Risk Prediction and Management}

With the increase in the suicide rate, particularly among the youths, researchers and clinicians have explored the utility of Artificial Intelligence (AI) in suicidal risk prediction and management [27] have conducted a general review of the scholarly works on the utility of $\mathrm{AI}$ in suicidal risk prediction and management. According to these authors, several studies have been directed toward the role of $\mathrm{AI}$ in identifying suicidal risk and clinical factors such as state of depression, past clinical diagnosis, substance abuse, and treatment history $[28,29]$ while other studies have explored the environmental factors [30,31].

The starting point for most of such studies is Machine Learning (ML), depending on the availability of data, to do modeling and potentially prediction. A key challenge in this nascent field is access to adequate and appropriate data. According to [27] though suicidal risk factors have been generally identified, data has not yet been fully integrated into a reliable predictive model. The clinical process of identifying risk factors and other related diagnosis are still very subjective and varies among therapists and clinicians. As a result, common statistical analytical approaches, which are generally limited in analyzing complex data, have not so far been successful in predicting suicidal behaviors [32].

As the volume and variety of clinical data are increasing significantly, there is hope that AI can help with potential prediction of risk factors to supplement clinical diagnosis. Some studies and exploratory analyses have shown early promises in 
identifying predominant suicide risk domains. These works have identified clinical risk, such as sudden mood change, recent history of low mood, self-injury, psychiatric comorbidity and previous hospitalizations $[33,29]$ and cognitive risk, such as sentiments around life satisfaction, purpose, hopelessness, self-esteem and selfperceived competency [34,35] Sentiment analysis is one area with potential promise for suicidal risk prediction and management.

Another AI-related area that has received quite a bit of attention recently is the so called 'conversational agents'. Conversational agents utilize Natural Language Processing (NLP) by simulating conversations with individuals and generating human-like responses using a text- or voice-based connectivity [36] This new approach has been increasingly adopted by therapists and clinicians to deliver psychological interventions such as social therapy [37] cognitive behavioral therapy [38] and trauma therapy [39]. With the increase use of devices such as smartphones, intervention delivery can be provided to a patient's emotional state [37]. Though AI-based approaches to suicide risk prediction and management are still in their early stage, the advancement in technology and clinical processes, such as better clinical data integration and sentiment analysis, can perhaps enhance the current prediction and prevention attempts, to identify risk factors in real time and potentially allow for mitigation of the increasing likelihood of suicides among the youths.

\section{Concluding Remarks}

Globally, suicide is an epidemic and a dangerous silent disease among individuals 14 to 28 years old and is rising at an alarming annual rate in the United States. Youngsters are often resorting to suicide due to severe depression and the current efforts to predict the risk factors and mitigate them are falling short. Modern electronic-based interactions, such as virtual socialization(esocialization), texting, and social media, have often replaced simple human interactions and dialogue such as 'Hello, How Are You' among most members of this demographic. The proliferation of electronic devices such as iPod, iPad, smart phones and any personal computers or electronic game platforms appears to have contributed to the seclusion of this vulnerable group, further diminishing personal interactions that can possibly mitigate the increasing rate. Young individuals nowadays do not often socialize and interact with family members and the community at large as much as our previous generations did. Lack of daily routine personal interactions appears to add to the severity of depression in particular among the youth.

Moreover, pharmacological treatments of depression via medications or an invasive treatment such as Electroconvulsive Therapy (ECT) do not appear to be highly effective for Major Depressive Disorder (MDD). The first author of this article recommends other methodology such as Transcranial Magnetic Stimulation (TMS) or repetitive TMS (RTMS) as alternative and non-invasive approaches [2,3]. Recent studies, however, underline that both the size and complexity of data collected from health institutions, including patient records, images, and test results. The growing volume and variety of data can provide a basis for better analyses leading to better diagnosis and prognosis. AIbased approaches, integrating medicine and data science, have the potential to supplement the current efforts at suicide risk factors identification and management, as well as intervention delivery [40-42].

\section{References}

1. Jean Whalen (2018) Youth Suicidal Behavior Is on the Rise, Especially Among Girls. The Wall Street Journal, USA.

2. Bahman Zohuri, Patrick J McDaniel (2019) Electrical Brain Stimulation for the Treatment of Neurological Disorders. Apple Academic Press and CRC Press (CRC) Taylor \& Francis Group pp. 550.

3. Bahman Zohuri, David R Modisette (2019) Electrical Brain Stimulation to Treat Neurological Disorder Journal of Health Science 7: 123-128.

4. Christian Nordqvist, https://www.medicalnewstoday.com/ articles/154543.php, UK.

5. Yonas G, Jung R (2008) Fixing brains. Innovation: America's Journal of Technology Commercialization 6(3).

6. Wassermann EM (1998) Risk and Safety of Repetitive Transcranial Magnetic Stimulation: Report and Recommendations from the International Workshop on the Safety of Repetitive Transcranial Magnetic Stimulation, June 5-7, 1996. Electroenceph. Clin. Neurophysiol. 108(1): $1-16$

7. https://medlineplus.gov/depression.html, USA.

8. https://www.wsj.com/articles/cdc-finds-rise-in-suicide-rates-acrossthe-u-s-1528417378?mod=article_inline

9. Bertolote JM (2001) Suicide in the world: an epidemiological overview, 1959-2000. In: Wasserman D editor. Suicide - an unnecessary death. London: Dunitz, UK pp: 3-10.

10. Wasserman D (2001) Suicide- an unnecessary death. London pp: 160.

11. La Vecchia C, Lucchini F, Levi F (1994) Worldwide trends in suicide mortality, 1955-1989. Acta Psychiatr Scand 90(1): 53-64.

12. Diekstra RFW (1996) The epidemiology of suicide and parasuicide. Arch Suicide Res 2(1): 1-29.

13. Schmidtke A, Weinracker B, Apter A, Batt A, Berman A, et al. (1999) Suicide rates in the world: update. Arch Suicide Res 5(1): 81-89.

14. Yang B, Lester D (2004) Natural suicide rates in nations of the world. Crisis 25(4): 187-188.

15. https://www.nimh.nih.gov/health/topics/index.shtml

16. Lefaucheur JP (2009) Methods of therapeutic cortical stimulation. Neurophysiologie Clinique/Clinical Neurophysiology 39(1): 1-14.

17.http://www.scholarpedia.org/article/Transcranial_magnetic_ stimulation

18. Priori A (2003) Brain polarization in humans: a reappraisal of an old tool for prolonged non-invasive modulation of brain excitability. Clinical Neurophysiology 114(4): 589-595.

19. Yonas G, R Jung (2008) Fixing brains Innovation: America's journal of Technology Commercialization 6(3).

20. Ujkaj M, Davidoff DA (2012) A novel approach to individualized treatment in psychiatry: use of neuroimaging to predict efficacy and tolerability of ECT treatment for depression in routine clinical practice. Journal of ECT 28(2): 149-149.

21.Zaghi S, Acar M, Hultgren B, Boggio PS, Fregni F (2010) Noninvasive brain stimulation with low-intensity electrical currents: putative 
mechanisms of action for direct and alternating current stimulation. The Neuroscientist 16(3): 285-307.

22. Vera Moliadze, Andrea Antal, Walter Paulus (2010) Boosting brain excitability by transcranial high frequency stimulation in the ripple range. journal of Physiology 588(24): 4891-4904.

23. Schoen I, Fromherz P (2008) Extracellular stimulation of mammalian neurons through repetitive activation of $\mathrm{Na}+$ channels by weak capacitive currents on a silicon chip. I. Neurophvsiol 100(1): 346-357.

24. Terney D, Chaieb L, Moliadze V, Antal A, Paulus W, et al. (2008) Increasing human brain excitability by transcranial high-frequency random noise stimulation. Journal of Neuroscience 28(52): 14147-14155.

25. Fertonani A, Pirulli C, Miniussi C, (2011) Random noise stimulation improves neuroplasticity in perceptual learning. journal of Neuroscience 31(43): 15416-15423.

26. Mulquiney, PG, Hoy KE, Daskalakis ZJ, Fitzgerald PB (2011) Improving working memory: Exploring the effect of transcranial random noise stimulation and transcranial direct current stimulation on the dorsolateral prefrontal cortex. Clinical Neurophysiology 122(12): 23842389.

27. Trehani M Fonseka, Venkat Bhat, Sidney H Kennedy (2019) The utility of artificial intelligence in suicide risk prediction and the management of suicidal behaviors. Australian \& New Zealand Journal of Psychiatry 53(10): 954-964

28. Jenkins AL, Singer J, Conner BT, Shawna Calhoun MPH, Guy Diamond (2014) Risk for suicidal ideation and attempt among a primary care sample of adolescents engaging in nonsuicidal self-injury. Suicide and Life-Threatening Behavior 44(6): 616-628.

29. Passos IC, Mwangi B, Cao B, Hamilton JE, Wu MJ, et al. (2016) Identifying a clinical signature of suicidality among patients with mood disorders: A pilot study using a machine learning approach. Journal of Affective Disorders 193: 109-116.

30. Bonner RL, Rich AR (1990) Psychosocial vulnerability, life stress, and suicide ideation in a jail population: A cross-validation study. Suicide and Life Threatening Behavior 20(3): 213-224.

31. Fernandez-Arteaga V, Tovilla-Zarate CA, Fresan A, González-Castro TB, Juárez-Rojop IE, et al. (2016) Association between completed suicide and environmental temperature in a Mexican population, using the knowledge discovery in database approach. Comput Methods Programs Biomed 135: 219-224.
32. Franklin JC, Ribeiro JD, Fox KR, Bentley KH, Kleiman EM, et al. (2017) Risk factors for suicidal thoughts and behaviors: A meta-analysis of 50 years of research. Psychological Bulletin 143(2): 187-232.

33. Liu Y, Sareen J, Bolton JM, Wang JL (2016) Development and validation of a risk prediction algorithm for the recurrence of suicidal ideation among general population with low mood. Journal of Affective Disorders 193: 11-17.

34. Barros J, Morales S, Echavarri O, García A, Ortega J, et al. (2017) Suicide detection in Chile: Proposing a predictive model for suicide risk in a clinical sample of patients with mood disorders. Revista Brasileira de Psiquiatria 39(1): 1-11.

35. Jordan P, Shedden-Mora MC, Lowe B (2018) Predicting suicidal ideation in primary care: An approach to identify easily assessable key variables. General Hospital Psychiatry 51: 106-111.

36. Dahiya M (2017) A tool of conversation: Chatbot. International Journal of Computer Sciences and Engineering 5(5): 158-161.

37. D’Alfonso S, Santesteban-Echarri O, Rice S, Wadley G, Lederman R, et al. (2017) Artificial intelligence-assisted online social therapy for youth mental health. Frontiers in Psychology 8: PP796.

38. Fitzpatrick KK, Darcy A, Vierhile M (2017) Delivering cognitive behavior therapy to young adults with symptoms of depression and anxiety using a fully automated conversational agent (Woebot): A randomized controlled trial. JMIR Mental Health 4(2): e19.

39. Tielman ML, Neerincx MA, Bidarra R, Kybartas B, Brinkman WP (2017) A therapy system for post-traumatic stress disorder using a virtual agent and virtual storytelling to reconstruct traumatic memories. Journal of Medical Systems 41(8): PP125.

40. Turker Teking Erguzel, Nevzat Tarhan (2016) Machine Learning Approaches to Predict Repetitive Transcranial Magnetic Stimulation Treatment Response in Major Disorder. SAI Intelligent System Conference, London, United Kingdom PP: 1-6.

41. Danuta Wasserman, QI Cheng, Guo-Xin Jiang (2005) Global suicide rates among young people aged 15-19. World Psychiatry 4(2): 114-120.

42. Wassermann EM (1998) Risk and safety of repetitive transcranial magnetic stimulation: report and recommendations from the international workshop on the safety of repetitive transcranial magnetic stimulation, June 5-7,1996. Electroenceph clin Neurophysiol 108(1): 1-16.

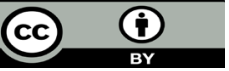

This work is licensed under Creative Commons Attribution 4.0 License

To Submit Your Article Click Here:

Submit Article

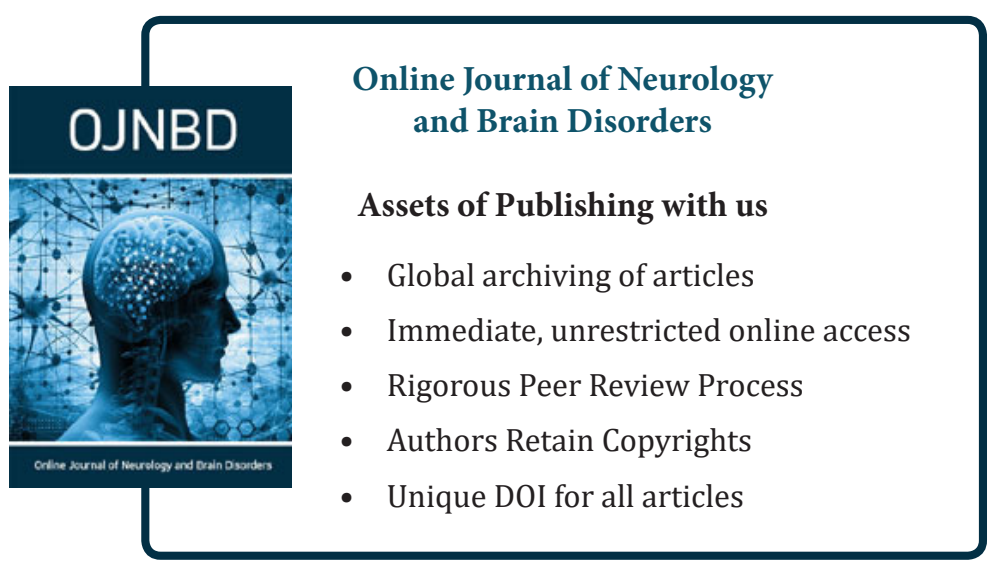

